\title{
DIGITAL PRODUCTION CONTROL OF MANUFACTURING WORKSHOP BASED ON INTERNET OF THINGS
}

\author{
Wang, N.*; Li, X. J. ${ }^{* * \#} \&$ Nie, H.* \\ * School of Management, Shandong Technology and Business University, Yantai 264005, China \\ ${ }^{* *}$ School of Business Administration, Hunan University of Finance and Economics, \\ Changsha 410205, China \\ E-Mail: wangning114@sdtbu.edu.cn, lixiaojuan@hufe.edu.cn, 2020410084@sdtbu.edu.cn
}

$\left({ }^{\#}\right.$ Corresponding author)

\begin{abstract}
The penetration of Internet of things (IoT) in the production control of modern manufacturing workshop has prompted advanced intelligent and information-based manufacturing techniques. However, the relevant studies fail to collect and process the various manufacturing parameters in real time, and rarely consider the coordination between manufacturing workshop and machines. This paper explores the digital production control of manufacturing workshop based on the IoT. Firstly, block diagrams were drawn for IoT-based digital workshop production control and software design of digital production control system, and a multi-objective optimization model was established for IoT-based digital workshop production control, aiming to optimize delivery time, machine load, and machine utilization rate. Next, the order sequencing method was detailed for digital workshop production control. Finally, machine competency and collaborative efficiency were introduced as optimization objectives, and an IoT-based strategy was designed for collaborative allocation of sub-tasks for workshop production. Our method was proved effective through experiments.

(Received in July 2021, accepted in August 2021. This paper was with the authors 3 weeks for 1 revision.)
\end{abstract}

Key Words: Internet of Things (IoT), Digital Production, Production Control, Manufacturing Workshop

\section{INTRODUCTION}

In the past two decades, China has achieved a rapid progress of manufacturing. But there is still a large gap from the advanced level of manufacturing in developed countries [1-7]. Since the proposal of the China Manufacturing 2025 strategy, the penetration of Internet of Things (IoT) in the production control of modern manufacturing workshop has prompted advanced intelligent and information-based manufacturing techniques, such as manufacturing logistics [8-11]. Combined with wireless sensor network (WSN) and swarm intelligence autonomous perception, these emerging techniques support the whole-process perception of intelligent manufacturing, and provide data support to the rational allocation of production resources and anomaly detection [12-14].

Digital information technology effectively improves the collaborative production capacity of various production units in the manufacturing industry [15-17]. Pabst et al. [18] applied the IoT technology to optimize the control system of intelligent production units, and realize the control of flexible production of various products in small batches. To ensure the production safety of energetic materials and mitigate the risks of the production site, Eriksson et al. [19] proposed an IoT-based digital monitoring method for production machines to replace manual patrol, and achieved such functions as real-time safety data collection, safety evaluation of production process and scheduling safety, and prewarning of production process risks. Cunbo et al. [20] summarized the IoT demand arising from common production anomalies in intelligent manufacturing, and constructed an IoT-based monitoring mode and numerical simulation model for production anomalies, which can process complex production tasks and predict the production state in real time. To quickly cope with various production anomalies, 
Renzi et al. [21] designed an IoT-based machine state monitoring system for data collection and monitoring of the packaging industry, and forecasted the quality of various products robustly based on XGBoost algorithm. Considering the current demand of intelligent manufacturing for a sound information management system, Lim et al. [22] developed an IoTbased real-time monitoring system for agricultural machinery production lines, and grasped the real-time changes in the performance and faults of production lines, and in the process environment of the workshop. In this way, Lim et al. [22] managed to allocate resources reasonably, improve the quality of agricultural machinery effectively, and control the production risks.

The development of digital production control has revealed multiple attributes of the workshop manufacturing process, such as stochasticity, dynamicity, and uncertainty. On the production control of order-driven manufacturing workshop, the relevant studies fail to collect and process the various manufacturing parameters in real time, and rarely consider the coordination between manufacturing workshop and machines. To solve the problems, this paper introduces the IoT technology to the digital production control of manufacturing workshop, and carries out a series of analyses. Section 2 presents the block diagrams for IoT-based digital workshop production control and software design of digital production control system, and establishes a multi-objective optimization model for IoT-based digital workshop production control, which has three optimization objectives: delivery time, machine load, and machine utilization ratio. Section 3 realizes the order sequencing for digital workshop production control. Section 4 provides an IoT-based sub-task collaborative allocation strategy or workshop production process. The proposed production control strategy and solving method were proved effective through experiments.

\section{IOT-BASED DIGITAL WORKSHOP PRODUCTION CONTROL} MODEL

To realize digital workshop production control, the massive data in the workshop need to be collected and processed in real time. The IoT and advanced production control strategies lay the basis for collecting, transmitting, processing, and systematically responding to the data of workshop IoT. Fig. 1 provides the block diagram for IoT-based digital workshop production control.

As shown in Fig. 1, the IoT-based digital workshop production control system mainly consists of four subsystems, namely, top management, digital production management, production resources, and production means. The top management subsystem mainly comprises an Internet-based visual monitoring platform and a server cloud platform. The digital production management subsystem is responsible for processing the manufacturing information and requirements, and decomposing and allocating sub-tasks. The production resources subsystem includes the machines, mobile platforms, and smart delivery of intelligent terminals and wireless sensors. The production means subsystem connects production means like operators and jobs with various production resources.

Fig. 2 presents the block diagram for software design of digital production control system. On the lower layer of the system, the data packets transmitted determine the types and requirements of the multi-threaded task of machines. The workshop layer involves local database of machines, multi-objective production control model, collaborative task distribution algorithm, communication protocol of production entities, workshop level application, and HCI interface. The top layer consists of digital production control system database, visual application, Internet application, and network interfaces. 


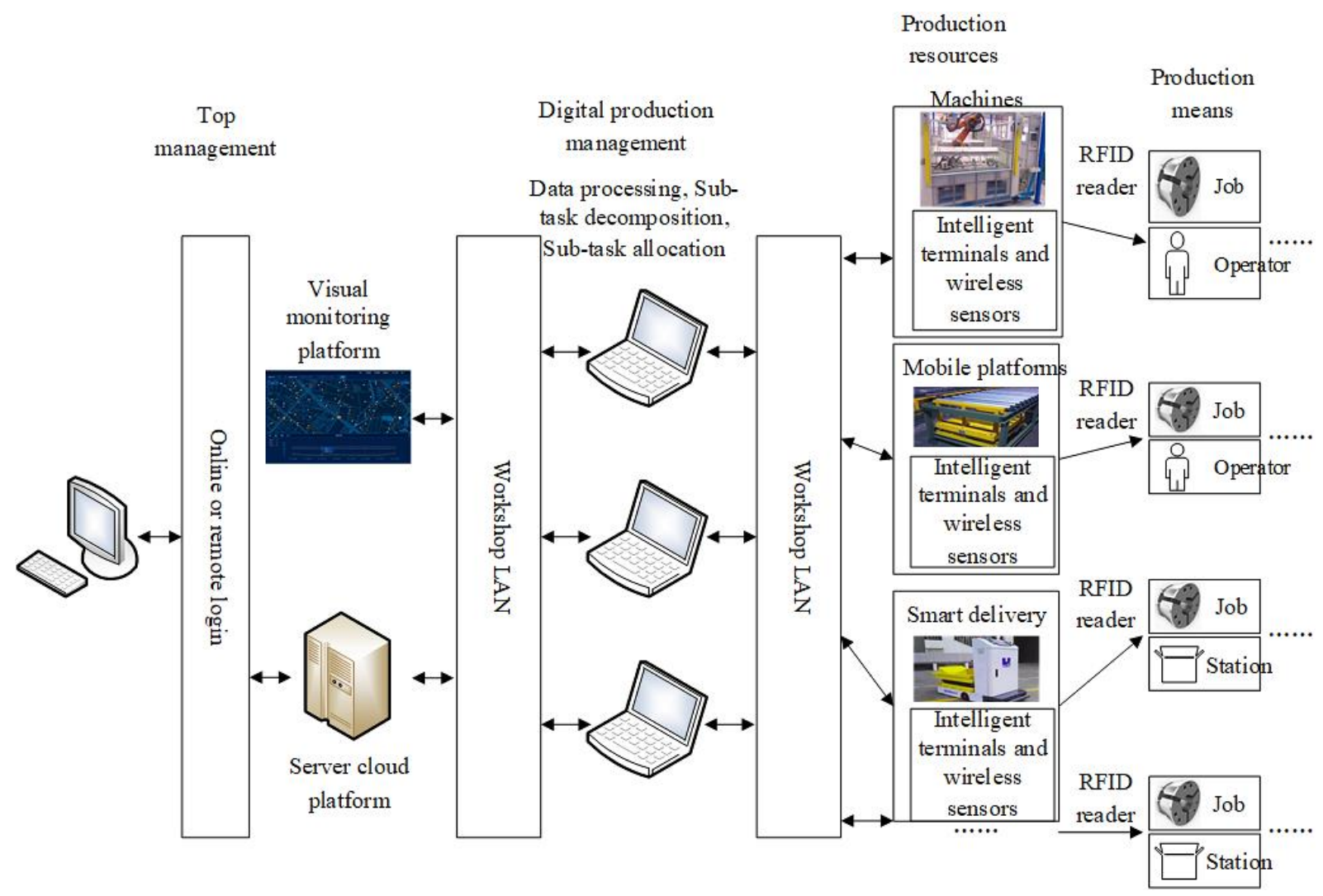

Figure 1: Block diagram for IoT-based digital workshop production control.

Note: LAN and RFID are short for local area network and radio-frequency identification, respectively.

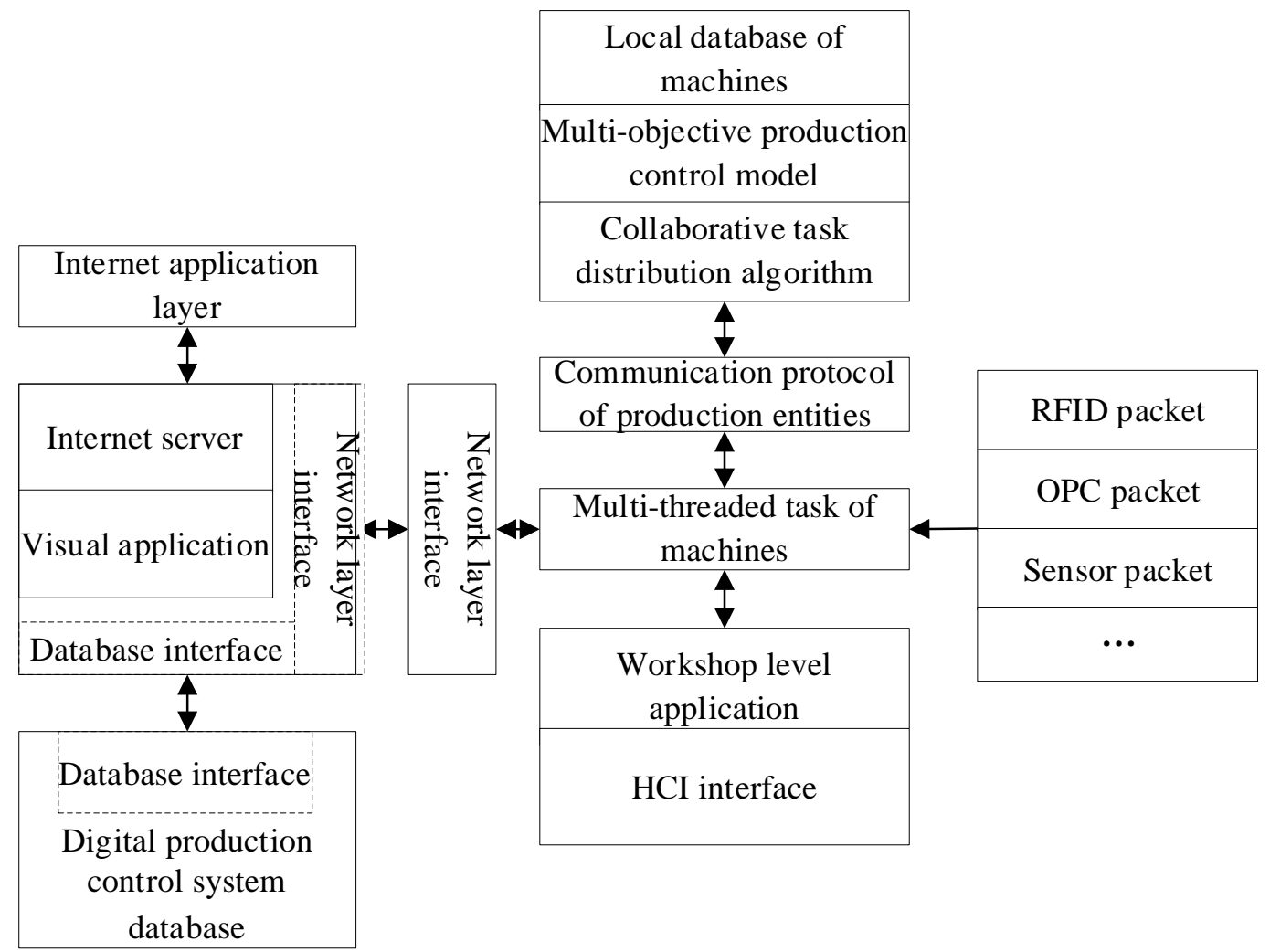

Figure 2: Block diagram for software design of digital production control system.

Note: $\mathrm{HCI}$ and OPC are short for human-computer interaction and open platform communication, respectively. 
The digital production control of a production task in the manufacturing workshop must fully consider the utilization rate of all machines and the load balance of the entire workshop. Under the premise that all machines in the workshop have the same load, the most efficient machine should be selected to shorten the production cycle and reduce the energy consumption of the production task. Therefore, this paper sets up a multi-objective digital workshop production control model, with delivery time, machine load, and machine utilization rate as the optimization objectives.

The set of jobs is denoted as $\psi=\left\{\tau_{1}, \tau_{2}, \ldots, \tau_{M e}\right\}$, where $\tau_{i}$ is the $i^{\text {th }}$ job among the $M_{e}$ jobs. Each job needs to go through multiple operations $W G_{i}=\left\{P_{i 1}, P_{i 2}, \ldots, P_{i M i}\right\}$, where $P_{i j}$ is the $j^{\text {th }}$ operation among the $M_{i}$ operations of the $i^{\text {th }}$ job. The set of machines is denoted as $N=\left\{N_{1}, N_{2}\right.$, $\left.\ldots, N_{n}\right\}$, where $N \tau$ is the $\tau^{\text {th }}$ machine among the $n$ machines. The set of machine capacities is denoted as $h=\left\{h_{1}, h_{2}, \ldots, h_{m}\right\}$. The $m_{i}$ machines with the capacity of $h_{d}$ are gathered into a set $h_{d}=\left\{N_{R}, N_{S}, \ldots, N_{T}\right\}$. Let $\left(P_{i j}, N_{\tau}\right)$ denote that the $j^{\text {th }}$ operation of the $i^{\text {th }}$ job will be processed on machine $N \tau$, and $W H\left(P_{i j}, N_{\tau}\right)$ be the corresponding preparation time for resetting, calibration, tool change, and cleaning. The start time and processing time of $\left(P_{i j}, N_{\tau}\right)$ are denoted as $W U\left(P_{i j}, N_{\tau}\right)$ and $W G\left(P_{i j}, N_{\tau}\right)$, respectively. The waiting time of $P_{i j}$ in the buffer zone of a machine is denoted as $B U\left(P_{i j}, N_{\tau}\right)$.

Then, the operation sequence of a job needs to satisfy:

$$
W U\left(P_{i j}, N_{n}\right)-W U\left(P_{i j}, N_{m}\right)+W G\left(P_{i j}, N_{n}\right) \leq 0
$$

One operation can only be processed on one machine:

$$
\sum_{k=1}^{m} X_{i j k}=1 \sum_{l=1}^{n} A_{i j l}=1
$$

where, $A_{i j l}$ is a binary function:

$$
\begin{aligned}
X_{i j k} & = \begin{cases}1 & \text { Operation } O_{i j k} \text { is processed on machine } M_{k} ; \\
0 & \text { Otherwise }\end{cases} \\
A_{i j l} & = \begin{cases}1 & \text { Operation } P_{i j l} \text { is processed on machine } N_{l} \\
0 & \text { Otherwise }\end{cases}
\end{aligned}
$$

As for the optimization objectives, the load $Q_{h}$ of machine $N_{h}$ is the total processing time of the jobs on that machine:

$$
Q_{h}=\sum_{i=1}^{M_{e}} \sum_{j=1}^{M_{i}} A_{i j l} W G\left(P_{i j}, N_{h}\right)
$$

Eq. (4) shows that $Q_{h}$ needs to be calculated based on the data collected in real time from the production control process. Let $\xi_{h}$ be the utilization rate of $N_{h}$. Then, the machine utilization rate can be characterized by the ratio the total time of $N_{h}$ being used for production to the total time of energization:

$$
\xi_{h}=\frac{Q_{h}}{\sum_{i=1}^{M_{e}} \sum_{j=1}^{M_{i}} A_{i j l} W H\left(P_{i j}, N_{h}\right)+W G\left(P_{i j}, N_{h}\right)+B U\left(P_{i j}, N_{\tau}\right)}
$$

Unlike $Q_{h}, \xi_{h}$ needs to be calculated based on the historical operation data of the machines in the workshop.

The energy efficiency of workshop production control can be evaluated by four types of indices: those to be minimized, those to be maximized, those to be controlled in an interval, and those to be controlled in the medium range. The digital workshop production control aims to minimize machine load $Q_{h}$ and delivery time $D E$, and maximize machine utilization rate $\xi_{h}$. That is, $Q_{h}$ and $D E$ should be minimized, while $\xi_{h}$ should be maximized. Since the evaluation indices for the energy efficiency of digital workshop production control belong to different types, all the indices must be converted to a uniform format before comprehensive evaluation. Therefore, $Q_{h}$ was uniformized by: 


$$
Q_{h}^{\prime}=\frac{1}{Q_{h}}
$$

The reciprocal of $D E$ was taken:

$$
D E^{\prime}=\frac{1}{D E}
$$

There are two key variables, namely, actual index value and evaluated index value, in the evaluation index system (EIS) for the energy efficiency of digital workshop production control. To convert between the two key variables, $Q_{h}, \xi_{h}$, and $D E$ were respectively normalized by:

$$
\begin{gathered}
L_{h}=\frac{Q_{h}^{\prime}-Q_{h \min }^{\prime}}{Q_{h \max }^{\prime}-Q_{h \min }^{\prime}} \\
W_{h}=\frac{\xi_{h}-\xi_{h \min }}{\xi_{h \max }-\xi_{h \min }} \\
D E_{h}=\frac{D E^{\prime}-D E_{\text {min }}^{\prime}}{D E_{\text {max }}^{\prime}-D E_{\text {min }}^{\prime}}
\end{gathered}
$$

The final comprehensive evaluation score can be obtained by summing up the values of the multiple indices in the said EIS. Let $a_{i}$ and $\theta_{i}$ be the evaluation value and weight coefficient of each index. Then, the final evaluation score $a$ of the energy efficiency of digital workshop production control can be expressed as:

$$
a=\prod_{i=1}^{m} a_{i}^{\theta_{i}}
$$

The summation approach in Eq. (11) is sensitive to the changes of a single index value. Since machine load and machine utilization rate are correlated, and independent of delivery time, the final evaluation score was derived by summing up the indices through both addition and multiplication:

$$
V_{h}=W_{h} L_{h}+D E_{h}
$$

\section{ORDER SEQUENCING}

In the digital production control system for order-driven workshop, the production objectives, such as the time of order placement, demand of product quality, and delivery period, vary with the orders. The digital production control system should fully consider the material situation, order features, and machine capacity, and maximize the production benefit through IoT-based control. Therefore, the orders in the task queue should be sorted by priority from three aspects: task allocation, delivery task selection, and machine selection. This paper adopts fuzzy comprehensive evaluation (FCE) to sort the orders by priority, under the effects of both quantitative and qualitative factors. Fig. 3 presents the block diagram of order sequencing.

This paper considers the following factors affecting order priority: order value, delivery period, client importance, type of client, and technical difficulty. Each factor was evaluated against a 5-level scale. The five levels are excellent, good, medium, qualified, unqualified. The evaluation scores of the five levels are $10,8,6,4$, and 2 , respectively. The corresponding judgment set can be expressed as $J U=\left\{o_{1}, o_{2}, o_{3}, o_{4}, o_{5}\right\}$, where $J U_{i}$ is the evaluation score of the $i^{\text {th }}$ level in $J U$. The index weights $\omega=\left(\omega_{1}, \omega_{2}, \omega_{3}, \omega_{4}\right\}$ were solved through analytic hierarchy process (AHP), under the premise that $\omega_{i}$ is greater than 0 and the sum of all $\omega_{i}$ equals 1 . 


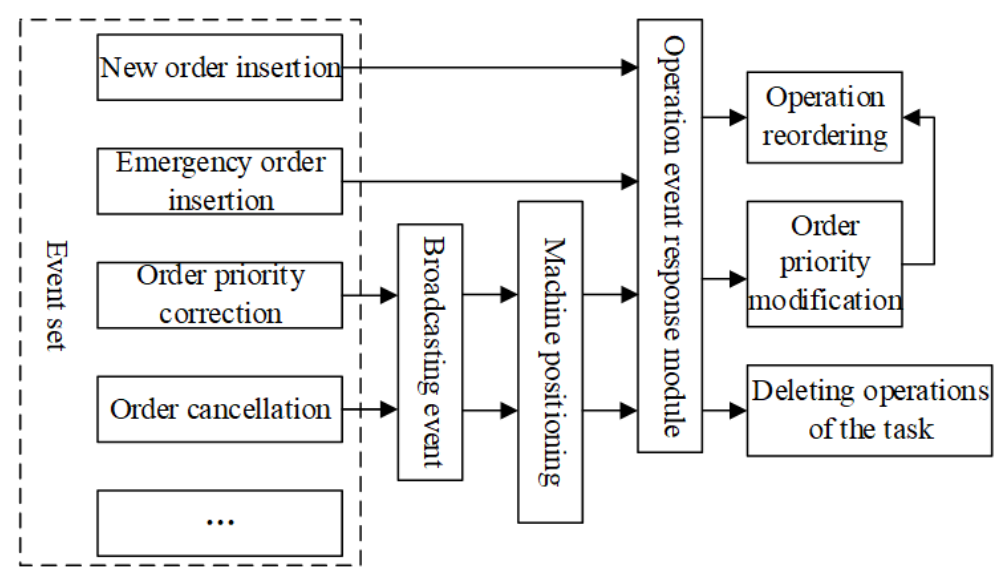

Figure 3: Block diagram of order sequencing.

Let $r i \varphi_{i}$ be the single factor evaluation matrix of order sequencing in digital workshop production control; $\varphi_{i j}$ be the proportion of experts selecting the $j^{\text {th }}$ element of the $i^{\text {th }}$ index in $J U$. Then, we have:

$$
\varphi_{i}=\left(\varphi_{i 1}, \varphi_{i 2}, \varphi_{i 3}, \varphi_{i 4}, \varphi_{i 5}\right)
$$

After the evaluation, the fuzzy relation matrix FU can be expressed as:

$$
F U=\left(\begin{array}{lllll}
\varphi_{11} & \varphi_{12} & \varphi_{13} & \varphi_{14} & \varphi_{15} \\
\varphi_{21} & \varphi_{22} & \varphi_{23} & \varphi_{24} & \varphi_{25} \\
\varphi_{31} & \varphi_{32} & \varphi_{33} & \varphi_{34} & \varphi_{35} \\
\varphi_{41} & \varphi_{42} & \varphi_{43} & \varphi_{44} & \varphi_{45}
\end{array}\right)
$$

Let $N_{I}$ be the number of indices. Then, $\varphi_{i j}$ needs to satisfy:

$$
\sum_{j=1}^{m} \varphi_{i j}=1
$$

The membership of an index to a subset of the judgement set can be characterized by the row vector $R$ of the fuzzy evaluation scores of $F U$ :

$$
R=Q \cdot F U=\left(r_{1}, r_{2}, r_{3}, r_{4}\right)
$$

As a row vector, $R$ can only measure the membership of an order to a certain index value. However, the same evaluations might frequently occur due to the limited number of indices. To prevent this situation, a composite score $C O$ was designed to differentiate between the evaluation index values of different orders, and further clarify the priority level of each order:

$$
C O=\sum_{i=1}^{4} r_{i} o_{i}
$$

\section{COLLABORATIVE ALLOCATION OF SUB-TASKS}

During the allocation of production tasks, the IoT-based digital workshop production control system needs to assign the sub-tasks of each order to suitable machines by the order sequencing strategy mentioned in the preceding section, such that the task allocation scheme can maximize the production benefit and optimize product quality through digital production control. Based on our multi-objective digital workshop production control model, this section fully considers the fitness between sub-tasks and the workshop and its machines, the coordination between the workshop and machines, and the coordination efficiency between machines. Machine competency refers to how competent a machine is to execute the sub-task assigned to it. This parameter is affected by various factors of the machine, including production quality, load capacity, and production speed. Let $O_{h}(i, j)$ be the machine load; $V_{e}=\left(v_{i j}\right)_{n}{ }^{e} \times m$ be the production 
quality of the machine; $B$ be the level of the production quality; $U_{e}=\left(u_{i j}\right)_{n}{ }^{e} \times m$ be the production speed; $\gamma, \alpha$, and $\beta$ be weight coefficients; $c_{i j}$ be the competency of the $i^{\text {th }}$ machine for the $j^{\text {th }}$ subtask.

(1) Machine load

For a machine, the overall ability for its software and hardware to handle a task is denoted by coefficient $O_{h}(i, j)$. This ability is divided into four levels: $O_{h}(i, j)=4,3,2,1$ (excellent, good, ordinary, and poor).

(2) Production quality

The production quality of all machines in the digital production control system is described by a production quality matrix $V_{e}=\left(v_{i j}\right)_{n}{ }^{e} \times l^{e}$, where $v_{i j}$ is the production quality of the $j^{\text {th }}$ subtask on the $i^{\text {th }}$ machine. According to the production process and precision, $v_{i j}$ can be divided into six levels: $B=[0,2,4,6,8,10]$.

(3) Production speed

The matching between the speed for a machine to complete a sub-task and the ideal production cycle is described by $u_{i j}\left(0<u_{i j}<1\right)$.

The machine competency matrix for an order can be expressed as:

$$
C=\left[\begin{array}{ccc}
c_{11} & \cdots & c_{1 l} \\
\vdots & c_{i j} & \vdots \\
c_{n 1} & \vdots & c_{n l}
\end{array}\right]
$$

where, $c_{i j}$ can be calculated by:

$$
\begin{gathered}
z_{i j}=\alpha \times f(i, j)+\beta \times u_{i j}+\gamma \times v_{i j} \\
c_{i j}=\gamma \times O_{h}(i, j)+\alpha \times v_{i j}+\beta \times u_{i j}
\end{gathered}
$$

Collaborative efficiency quantifies the degree of collaboration between machines in the digital production control system. There are three components of the collaboration between machines: material component, information component, and sub-task component, which are respectively denoted as $\left(F L_{M}, F L_{I}, F L_{K}\right)$. In the context of the IoT, the collaborative levels of material component, information component, and sub-task component are measured respectively with cost, information response time, and order priority, which are respectively denoted as $\left(Z B_{M}, Z B_{I}, Z B_{K}\right)$. Then, the collaborative efficiency matrix of machines can be expressed as:

$$
\eta=\left[\begin{array}{ccc}
\eta_{11} & \cdots & \eta_{1 l} \\
\vdots & \eta_{i j} & \vdots \\
\eta_{n 1} & \vdots & \eta_{n l}
\end{array}\right]
$$

where, $\eta_{i j}$ can be calculated by:

$$
\eta_{i j}=F L_{M} \times Z B_{M}+F L_{I} \times Z B_{I}+F L_{K} \times Z B_{K}
$$

The collaborative components satisfy the following inequalities: $0 \leq F L_{M}, F L_{I}, F L_{K} \leq 1$, and $F L_{M}+F L_{I}+F L_{K} \leq 1$.

Let $v_{i j}$ be a binary decision variable. If $v_{i j}=0$, the $i^{\text {th }}$ sub-task is not assigned to the $j^{\text {th }}$ machine; if $v_{i j}=1$, the $i^{\text {th }}$ sub-task is assigned to the $j^{\text {th }}$ machine. The objective function of maximizing the overall machine competency can be defined as max $T A_{1}$. According to the allocation conditions for tasks in the digital workshop production control system, the optimal sub-task allocation model can be established to optimize both machine competency and collaborative efficiency under the basic constraints:

$$
\max T A_{1}=\sum_{i=1}^{l} \sum_{j=1}^{n} v_{i j} \cdot c_{i j}
$$

At least one machine should be assigned to each sub-task:

$$
\sum_{i=1}^{l} v_{i j} \geq 1
$$


The objective function of maximizing the collaborative efficiency can be defined as:

$$
\operatorname{MaxTA}_{2}=\sum_{i=1}^{l} \sum_{j=1}^{n} v_{i j} \cdot q_{i j}
$$

The maximum task execution time should fall within the allowable time range $\Phi$ :

$$
\operatorname{Max}\left(\tau_{1}, \tau_{2}, \ldots, \tau_{n}\right) \leq \Phi
$$

To total cost $C B_{T}$ of a task should fall within the workshop operation cost $C B_{M}{ }^{*}$ :

$$
C B_{T}=\sum_{i=1}^{l} P C_{i} \cdot \tau_{i} \leq C B_{M} *
$$

\section{MODEL SOLVING BY IMPROVED GENETIC ALGORITHM (GA)}

The traditional GA has two major defects: premature convergence and falling into local optimum. To overcome the defects, this paper improves the GA by redesigning the fitness function and genetic operators. Considering the actual situation of digital workshop production control, double layer coding was adopted to encode the operations and machines in the field of multi-objective digital workshop production control.

Table I: Time of assembly operations.

\begin{tabular}{|c|c|c|c|c|}
\hline \multirow{2}{*}{$I N$} & \multirow{2}{*}{$\Phi$} & \multicolumn{3}{|c|}{$N_{h}$} \\
\cline { 2 - 5 } & & $N_{h-1}$ & $N_{h-2}$ & $N_{h-3}$ \\
\hline \multirow{3}{*}{$I N_{1}$} & $\Phi_{11}$ & 3 & 6 & 2 \\
\cline { 2 - 5 } & $\Phi_{12}$ & 4 & 7 & 0 \\
\cline { 2 - 5 } & $\Phi_{13}$ & 0 & 1 & 5 \\
\hline \multirow{3}{*}{$I N_{2}$} & $\Phi_{21}$ & 2 & 5 & 0 \\
\cline { 2 - 5 } & $\Phi_{22}$ & 6 & 3 & 1 \\
\cline { 2 - 5 } & $\Phi_{23}$ & 2 & 4 & 0 \\
\hline \multirow{2}{*}{$I N_{3}$} & $\Phi_{31}$ & 7 & 1 & 2 \\
\cline { 2 - 5 } & $\Phi_{32}$ & 5 & 4 & 0 \\
\hline
\end{tabular}

Based on the actual situation in Table I, the task-operation correlation matrix IN and time matrix $\Phi$ can be respectively established as:

$$
\begin{aligned}
& {[1,2,3] \quad[1,2] \quad[2,3]} \\
& I N=[1,2] \quad[1,2,3] \quad[1,2] \\
& {[1,2,3] \quad[1,2] \quad[0]} \\
& {[3,6,2] \quad[4,7] \quad[1,5]} \\
& \Phi=[2,5] \quad[6,3,1] \quad[2,4] \\
& {[7,1,2] \quad[5,4] \quad[0]}
\end{aligned}
$$

Fig. 4 provides an example of the double layer coding method.

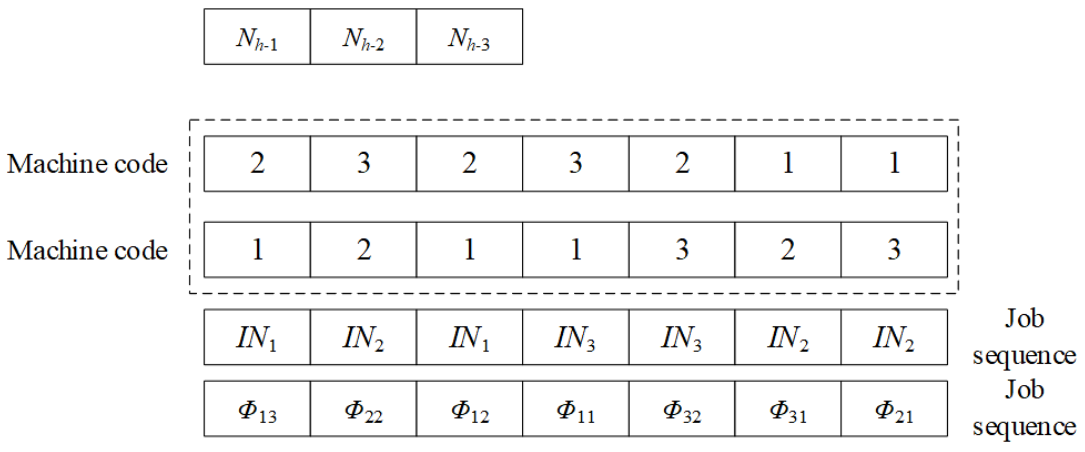

Figure 4: Example of double layer coding. 
As for decoding, the operations of the manufacturing process are decoded first. Following the above encoding rule, the chromosome genes are converted into the corresponding operations $G W_{j l}$. Based on $I N$ and $\Phi$, the serial number of the machine corresponding to each operation $G W_{j l}$ is solved, as well as the time needed to execute the operation on that machine. In addition, the operations are sorted in descending order of priority.

In the traditional GA, proportional selection is used to choose chromosomes from the population: divide the fitness of the target chromosome with the overall fitness of the population, and rely on the ratio to judge whether to retain that chromosome. The probability of retaining the $i^{\text {th }}$ chromosome in a population of $N_{D}$ chromosomes can be calculated by:

$$
G V_{i}=\frac{\text { fitness }_{i}}{\sum_{i=1}^{N_{D}} \text { fitness }_{i}}
$$

The proportional selection might suppress the diversity of chromosomes, and drag down the search speed. To avoid these defects, this paper directly retains the best chromosome in the population, and copies it to the child generation. Let $P S$ be the population size; $R N$ be a random number between 0 and 1 . The $i^{\text {th }}$ chromosome would be copied if it satisfies:

$$
\frac{\sum_{j=1}^{i-1} \text { fitness }_{i}}{\sum_{j=1}^{P S} \text { fitness }_{i}}<R N \leq \frac{\sum_{j=1}^{i} \text { fitness }_{i}}{\sum_{j=1}^{P S} \text { fitness }_{i}}
$$

Fig. 5 shows the flow of the improved GA.

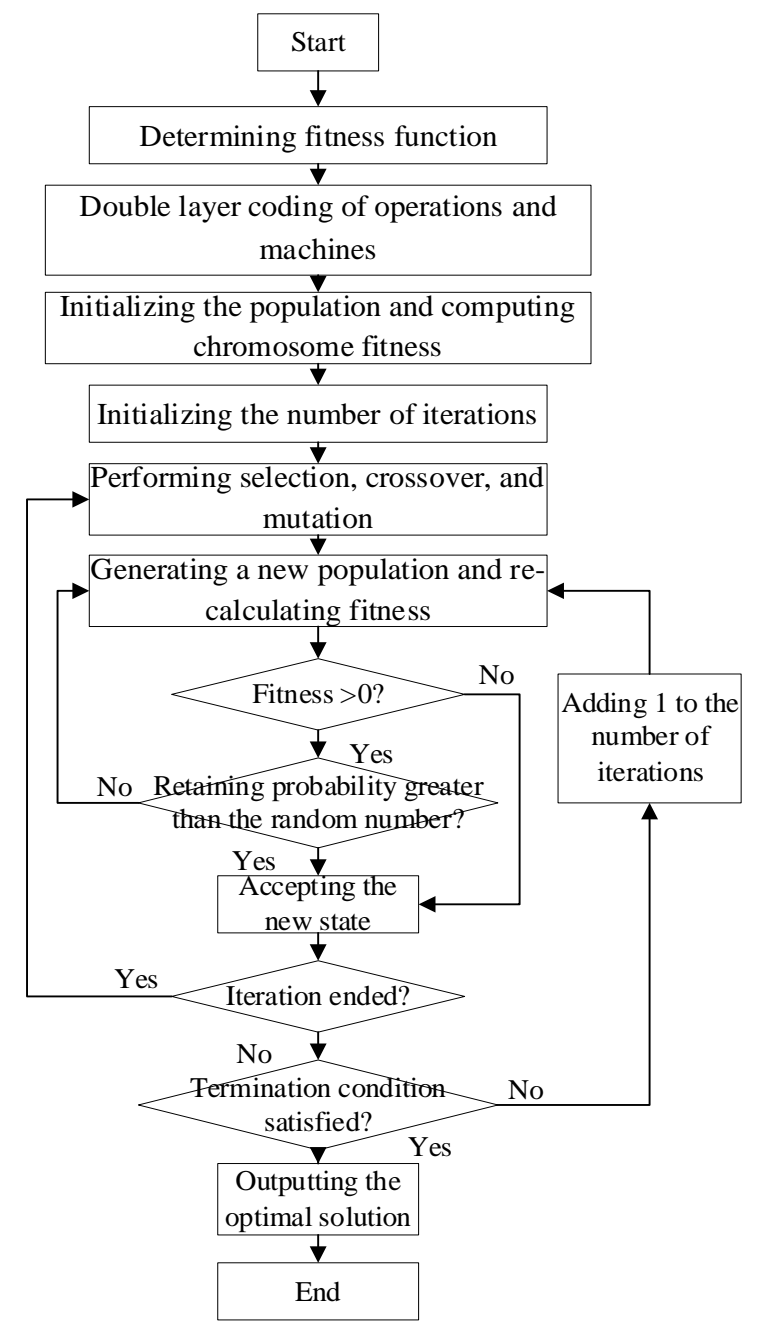

Figure 5: Flow of the improved GA. 


\section{EXPERIMENTS AND RESULTS ANALYSIS}

The solving results were not greatly affected by the changes of the parameters in the improved GA. Fig. 6 compares the convergence curves in 50 iterations of original and improved GA solving our digital production control model. The $\mathrm{x}$-axis represents the number of iterations, and the $y$-axis represents the error of the objective function. It is obvious that the improved GA achieved better convergence than the original algorithm.

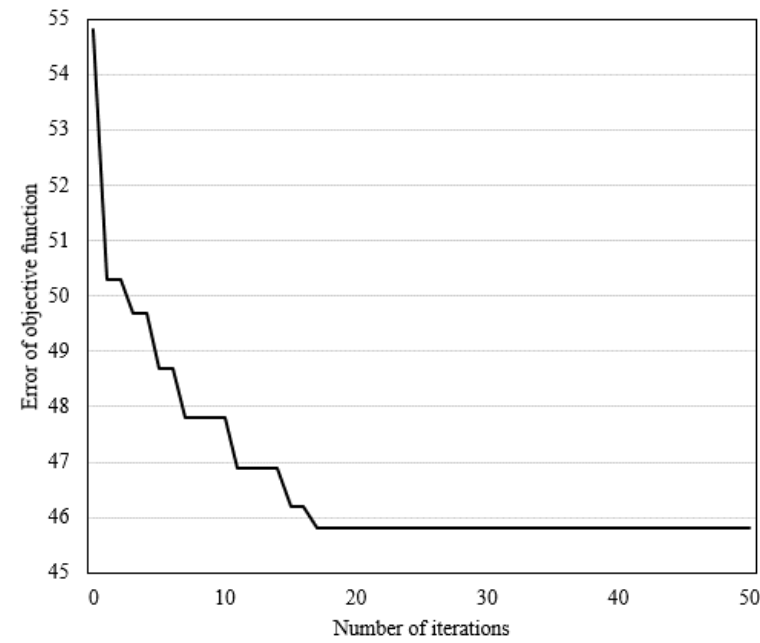

a) Original GA

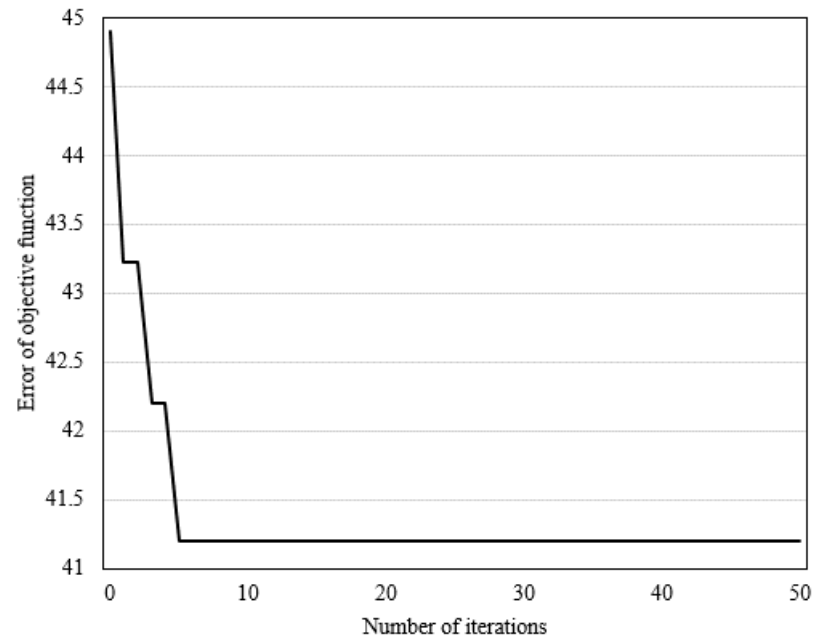

b) Improved GA

Figure 6: Convergence curves of original and improved GA solving our digital production control model.

Next, 35 simulations were conducted continuously. Fig. 7 compares the Gantt charts of the optimal solutions of the improved GA before and after the implementation of our digital production control scheme. The total production time was $26 \mathrm{~h}$, maximum machine load was 2 , mean algorithm runtime was $126 \mathrm{~s}$, and mean number of iterations was about 10 .

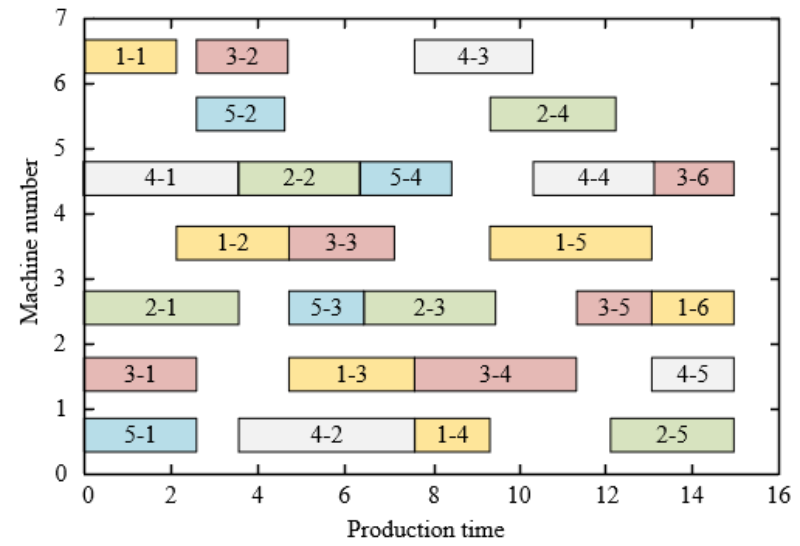

a) Pre-control

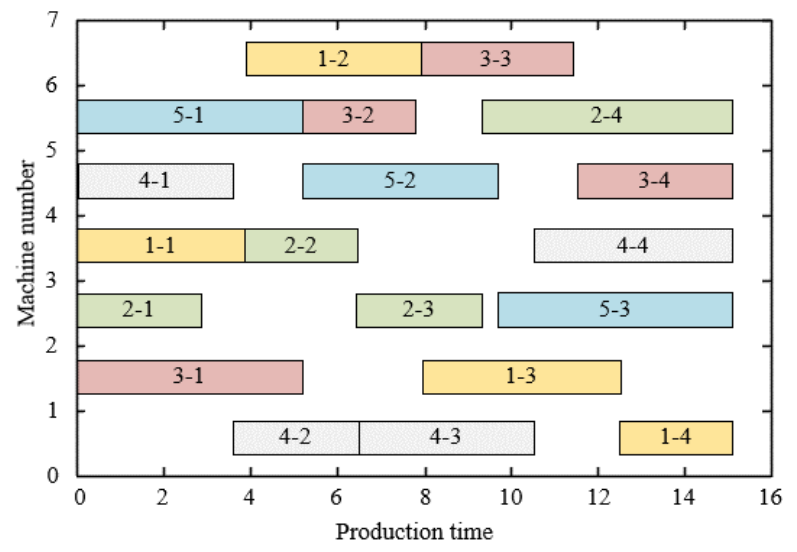

b) Post-control

Figure 7: Gantt charts of the optimal solutions of the improved GA before and after the implementation of our digital production control scheme.

Table II: Calculation effects of different algorithms.

\begin{tabular}{|c|c|c|c|}
\hline Algorithm & Processing time (h) & Maximum machine load & Mean runtime \\
\hline Traditional GA & 25 & 4 & 211 \\
\hline Improved fitness function & 22 & 5 & 189 \\
\hline Our algorithm & 18 & 3 & 83 \\
\hline
\end{tabular}


Our previous survey on the energy efficiency of IoT-based digital workshop production control systems shows that it generally takes 5-7 workdays to complete a complex processing and assembly task. Table II compares the calculation effects of different algorithms. It can be inferred that our improved GA solved the digital workshop production control model excellently, as evidenced by its stable results and high efficiency.

\section{CONCLUSIONS}

This paper probes deep into the digital production control of IoT-based workshop manufacturing. Firstly, a multi-objective IoT-based digital workshop production control model was constructed to optimize delivery time, machine load, and machine utilization rate. Then, the order sequencing method was detailed for digital workshop production control. Finally, machine competency and collaborative efficiency were introduced to develop an IoT-based strategy for collaborative allocation of sub-tasks for workshop production. Through experiments, the digital production control situation and scheme were determined for a realworld IoT-based digital workshop production control system, the convergence curves of original and improved GAs were drawn, and the Gantt charts of the improved GA were plotted before and after the implementation of our digital production control scheme. The experimental results confirm that our improved GA can solve the digital workshop production control model excellently, as evidenced by its stable results and high efficiency.

\section{ACKNOWLEDGEMENTS}

This paper was supported by National Social Science Foundation: a study on the behaviour of Crossorganizational knowledge sharing and its dynamic incentive Mechanism in the context of differentiation (20BGL126).

\section{REFERENCES}

[1] Ma, J.; Wang, Y.-C.; Zhao, M.; Jiang, Z.-Q.; E, M.-C.; Wang, Q. (2021). Visualization management and control methods of production cell based on digital twin, Computer Integrated Manufacturing Systems, Vol. 27, No. 5, 1256-1268, doi:10.13196/j.cims.2021.05.002

[2] Kutin, A.; Dolgov, V.; Sedykh, M. (2016). Information links between product life cycles and production system management in designing of digital manufacturing, Procedia CIRP, Vol. 41, 423-426, doi:10.1016/j.procir.2015.12.126

[3] Xu, S. Z. (2019). A petri net-based hybrid heuristic scheduling algorithm for flexible manufacturing system, International Journal of Simulation Modelling, Vol. 18, No. 2, 325-334, doi:10.2507/IJSIMM18(2)CO6

[4] Li, H.-Y.; Xu, W.; Cui, Y.; Wang, Z.; Xiao, M.; Sun, Z.-X. (2019). Preventive maintenance decision model of urban transportation system equipment based on multi-control units, IEEE Access, Vol. 8, 15851-15869, doi:10.1109/ACCESS.2019.2961433

[5] Awaga, A. L.; Xu, W.; Liu, L.; Zhang, Y. (2020). Evolutionary game of green manufacturing mode of enterprises under the influence of government reward and punishment, Advances in Production Engineering \& Management, Vol. 15, No. 4, 416-430, doi:10.14743/apem2020.4.375

[6] Zhang, Q.; Mu, R. Y.; Zhang, Z.; Hu, Y.; Liu, C. J.; Zhang, L.; Yu, X. (2020). Competitiveness evaluation of high-quality manufacturing development in the Yangtze river economic belt, International Journal of Sustainable Development and Planning, Vol. 15, No. 6, 875-883, doi:10.18280/ijsdp.150611

[7] Chen, L.; Ding, X.; Wu, X. (2015). Water management tool of industrial products: a case study of screen printing fabric and digital printing fabric, Ecological Indicators, Vol. 58, 86-94, doi: $10.1016 /$ j.ecolind.2015.05.045 
[8] Alexopoulos, K.; Makris, S.; Xanthakis, V.; Chryssolouris, G. (2011). A web-services oriented workflow management system for integrated digital production engineering, CIRP Journal of Manufacturing Science and Technology, Vol. 4, No. 3, 290-295, doi:10.1016/j.cirpj.2011.06.002

[9] He, Y. J. (2020). Influencing factors and evaluation model of quality risks in intelligent manufacturing mobile supply chain, Journal Européen des Systèmes Automatisés, Vol. 53, No. 6, 953-961, doi:10.18280/jesa.530621

[10] Belkadi, F.; Vidal, L. M.; Bernard, A.; Pei, E.; Sanfilippo, E. M. (2018). Towards an unified additive manufacturing product-process model for digital chain management purpose, Procedia CIRP, Vol. 70, 428-433, doi:10.1016/j.procir.2018.03.146

[11] Kozma, D.; Varga, P.; Soós, G. (2019). Supporting digital production, product lifecycle and supply chain management in Industry 4.0 by the arrowhead framework - a survey, 2019 IEEE $17^{\text {th }}$ International Conference on Industrial Informatics, 126-131, doi:10.1109/ INDIN41052.2019.8972216

[12] Altamiranda, E.; Colina, E. (2019). A system of systems digital twin to support life time management and life extension of subsea production systems, Proceedings of the OCEANS 2019Marseille, 9 pages, doi:10.1109/OCEANSE.2019.8867187

[13] Ma, J.; Chen, H.; Zhang, Y.; Guo, H.; Ren, Y.; Mo, R.; Liu, L. (2020). A digital twin-driven production management system for production workshop, The International Journal of Advanced Manufacturing Technology, Vol. 110, No. 5-6, 1385-1397, doi:10.1007/s00170-020-05977-5

[14] Ren, J.; Huang, S. Y.; Song, W.; Han, J. (2019). A novel indoor positioning algorithm for wireless sensor network based on received signal strength indicator filtering and improved Taylor series expansion, Traitement du Signal, Vol. 36, No. 1, 103-108, doi:10.18280/ts.360113

[15] Veynberg, R.; Koslova, M.; Askarov, G.; Bexultanov, A.; Yussupova, Z. (2020). Digital technologies in supply chain management in production, Proceedings of the $1^{\text {st }}$ International Conference on Business Technology for a Sustainable Environmental System, Paper 03006, 10 pages, doi:10.1051/e3sconf/202015903006

[16] Nazifa, T. H.; Ramachandran, K. K. (2019). Information sharing in supply chain management: A case study between the cooperative partners in manufacturing industry, Journal of System and Management Sciences, Vol. 9, No. 1, 19-47, doi:10.33168/JSMS.2019.0102

[17] Schuh, G.; Kelzenberg, C.; Wiese, J.; Ochel, T. (2019). Data structure of the digital shadow for systematic knowledge management systems in single and small batch production, Procedia CIRP, Vol. 84, 1094-1100, doi:10.1016/j.procir.2019.04.210

[18] Pabst, S.; Kim, H.; Polok, L.; Ila, V.; Waine, T.; Hilton, A.; Clifford, J. (2015). Jigsaw: multimodal big data management in digital film production, ACM SIGGRAPH 2015 Posters, Paper 50, 1 page, doi: $10.1145 / 2787626.2792617$

[19] Eriksson, H.; Sandberg, M.; Mukkavaara, J.; Jansson, G.; Stehn, L. (2019). Assessing digital information management between design and production in industrialised house-building - a case study, Proceedings of the $36^{\text {th }}$ International Symposium on Automation and Robotics in Construction, 340-347

[20] Cunbo, Z.; Liu, J.; Xiong, H. (2018). Digital twin-based smart production management and control framework for the complex product assembly shop-floor, The International Journal of Advanced Manufacturing Technology, Vol. 96, No. 1-4, 1149-1163, doi:10.1007/s00170-018-1617-6

[21] Renzi, D.; Maniar, D.; McNeill, S.; del Vecchio, C. (2017). Developing a digital twin for floating production systems integrity management, Proceedings of the 2017 Offshore Technology Conference, Paper OTC-28012-MS, 8 pages, doi:10.4043/28012-MS

[22] Lim, K. Y. H.; Zheng, P.; Chen, C.-H. (2019). A state-of-the-art survey of Digital Twin: techniques, engineering product lifecycle management and business innovation perspectives, Journal of Intelligent Manufacturing, Vol. 31, No. 6, 1313-1337, doi:10.1007/s10845-019-01512-w 